\title{
PEMERTAHANAN KEARIFAN LOKAL BASITA PARIBASA RING PUPULAN TEMBANG HITS SAKING DEK ULIK
}

\author{
Ni Pt. Rustiana Dewi ${ }^{1}$, I. A. Sukma Wirani ${ }^{1}$, I.A. Pt. Purnami ${ }^{2}$ \\ Jurusan Pendidikan Bahasa Bali \\ Universitas Pendidikan Ganesha \\ Singaraja, Indonesia
}

e-mail: \{itaputu2@gmail.com,sukma.wirani,putu.purnami\}@undiksha.ac.id

\section{KUUB}

Tetilik matetujon nlatarang: (1) kearifan lokal basita paribasa ring pupulan tembang hits Dek Ulik, (2) panampen yowana, Ian (3) panampen penyanyi lan pangawi tembang. Jejering tetilik: pupulan tembang hits Dek Ulik. Penandang tetilik: kearifan lokal basita paribasa. Tetilik nganggen kramaning dokumentasi, sadu wicara, lan kuesioner. Data tureksa sane kanggen: (1) reduksi data; (2) pangwedar data; lan (3) panyutetan (verifikasi). Pikolih tetilik pupulan tembang hits Dek Ulik, sakadi: (1) kearifan lokal basita paribasa sane kapanggihin wenten limang soroh, sakadi sasenggakan, wewangsalan, sesawangan, peparikan, lan sesonggan. (2) Para yowana ngicenin panampen sane becik ring kawentenan basita paribasa ring pupulan tembang Dek Ulik yadiastun basita paribasa meweh karesepang. (3) Panampen Dek Ulik pinaka penyanyi lan Raka Sidan pinaka pangawi sane uning lan ngresep tekening kawentenan basita paribasa ring tembangnyane, taler cumpu yening kawentenan punika kadadosang sinalih tunggil sarana ngajegang kearifan lokal basita paribasa.

Kruna jejaton: Kearifan Lokal, Paribasa, Tembang Bali

\section{ABSTRAK}

Penelitian ini bertujuan menjelaskan: (1) kearifan lokal basita paribasa di kumpulan lagu hits Dek Ulik, (2) pendapat anak muda, dan (3) pendapat penyanyi dan pencipta lagu. Subjek penelitian: kumpulan lagu hits Dek Ulik. Objek penelitian: kearifan lokal basita paribasa. Penelitian ini menggunakan metode dokumentasi, wawancara, dan kuesioner. Analisis data yang digunakan: (1) reduksi data; (2) deskripsi data; dan (3) kesimpulan/verifikasi. Hasil penelitian kumpulan lagu hits Dek Ulik, antara lain: (1) kearifan lokal basita paribasa yang ditemukan ada lima jenis, yaitu sasenggakan, wewangsalan, sesawangan, peparikan, dan sesonggan. (2) anak muda memberikan pendapat yang positif terhadap keberadaan basita paribasa di kumpulan lagu Dek Ulik walaupun basita paribasa sulit dipahami. (3) Dek Ulik sebagai penyanyi dan Raka Sidan sebagai pencipta lagu, mengetahui dan memahami keberadaan basita paribasa di lagu-lagunya, serta menyetujui apabila keberadaan tersebut dijadikan sebagai salah satu sarana untuk melestarikan kearifan lokal basita paribasa.

Kata kunci: Kearifan Lokal, Peribahasa, Lagu Bali 


\begin{abstract}
This study aims to explain: (1) local wisdom of basita paribasa in Dek Ulik's song collection, (2) the youth opinion, and (3) the opinion of the singer and the song's creator. Research subjects: Dek Ulik's song collection. The object of research: local wisdom of basita paribasa. The method of this research were documentation, interview, and questionnaire. Analysis of data used: (1) data reduction, (2) description of data, and (3) verification. The result of the research are: (1) the local wisdom of basita paribasa are found five kinds: sasenggakan, wewangsalan, sesawangan, peparikan, and sesonggan. (2) the youth opinion about local wisdom of basita paribasa are positive, although hard to understand it. (3) Dek Ulik as a singer and Raka Sidan as a song's creator know about the local wisdom of basita paribasa in their songs. They also agree if this existence can keep the local wisdom of basita paribasa.
\end{abstract}

Keywords: Local Wisdom, Proverb, Balinese' Song

\section{PURWAKA}

Basa daerah punika mabuat pisan ring daerah utawi wewilangannyane soang-soang. Basa daerah nyinahaang budaya lan identitas daerah. Basa punika prasida ngiusin kabudayaan (Chaer, 1994:70). Maka duaning asapunika, basa kabaos wagian saking budaya.

Basa Bali kadadosang tetamian saking panglingsir, sane kaaptiang pacang prasida ngajegang budaya Bali antuk makudang-kudang unsur utawi wangun sane ngwangun basa Bali. Sinalih tunggil wangun sane ngwangun basa Bali inggih punika basita paribasa. Ring soang-soang basa madue paribasa sane malianan, punika mawinan paribasa prasida nyihnayang identitas saking basa punika. Sakadi ring basa Bali madue basita paribasa sane ketah kaanggen panglengut basa ri kala magegonjakan lan ngicenin piteket sane kaanutang tekening kabudayaan Bali.

Basita paribasa ngranjing ring wagian kabudayaan Bali sane ketah kabaos kearifan lokal, mawinan panglimbak kearifan lokal prasida kapanggihin ring gegendingan, kidung, paribasa, tutur, semboyan, miwah kitabkitab kuno (Wikantiyoso lan Tutuko, 2009: 8). Kearifan lokal pinaka tetamian saking panglingsir sane panglimbaknyane kauningin majalaran antuk bebaosan utawi carita ring para krama. Kearifan lokal sane wenten ring daerah utawi wewilangan soang-soang punika majanten meweh kaajegang ring jagat aor tan pawates. Kawentenan basita paribasa pinaka kearifan lokal taler meweh pisan kaajegang. Sampun akidik krama Bali sane mabebaosan nganggen basita paribasa. Taler karasayang meweh ngresepang teges saking basita paribasa. Basa Bali taler silih sinunggil basa sane kaplajahin ring sekolah tur kawastanin plajahan muatan lokal. Punika nyinahaang ring sekolah-sekolah para sisia pastika sampun polih plajahan basa Bali lan kaicen pangajahan indik basita paribasa. Nanging kawentenane punika nenten ngranayang para sisia waged nguningin lan ngresepang basita paribasa sane pinaka kearifan lokal.

Krama Bali sapatutnyane eling tekening kearifan lokal basita paribasa. Mawinan basita paribasa prasida nyihnayang identitas Bali antuk basa. Kesusastraan Bali sane becik anggen sarana mlajahin basita paribasa inggih punika kasusastraan Bali tembang. Soroh tembang wenten sane purwa lan sane anyar. Soroh tembang purwa minakadi gegendingan, sekar alit, sekar madya, lan sekar agung. Soroh tembang Bali anyar minakadi tembang pop, rock, jazz, miwah sane lianan. Kawentenan basita paribasa ring tembang Bali purwa sujatinyane akeh, nanging pamilet utawi sane seneng tekening tembang Bali purwa akidik. Sangkaning asapunika, tembang Bali 
anyar pinaka sarana sane becik anggen mlajahin lan nguningin basita paribasa.

Sane mangkin ring Bali akeh krama Bali sane nguningayang daging pikenoh antuk kasusastraan marupa tembang sane kawastanin tembang pop Bali. Ring tembang Bali sakadi mangkin, kapanggihin madaging basita paribasa ring liriknyane. Kawentenan basita paribasa ring tembang pop Bali prasida kadadosang sarana nguningin basita paribasa. Krama Bali akeh sane uning lan seneng ring tembang pop Bali. Dadosnyane tembang pop Bali pinaka sarana mlajahin basita paribasa sane becik pisan taler nenten ngawinang rasa emed ri kala mlajahin.

Pinaka imba tembang pop Bali sane akeh kasenengin olih krama Bali inggih punika tembang-tembang saking Dek Ulik sane kapanggihin madaging basita paribasa ring liriknyane. Makehan tembang saking Dek Ulik ring soangsoang album punika stata dados hits utawi kasenengin mawinan prasida anut tekening kahuripan krama Bali. Kawentenan basita paribasa ring tembang-tembangnyane prasida kadadosang sarana anggen nguningin basita paribasa. Napi malih tembang Dek Ulik punika kasenengin olih krama Bali, pastika akeh sane miarsayang lan pacang kauningin kawentenan basita paribasa ring tembang punika malarapan antuk tetilikan puniki.

Bantang pikobet tetilik: (1) sapunapi kawentenan kearifan lokal basita paribasa ring pupulan tembang hits saking Dek Ulik? (2) sapunapi panampen saking para yowana indik kawentenan kearifan lokal basita paribasa ring pupulan tembang hits saking Dek Ulik? (3) sapunapi panampen saking penyanyi lan pangawi tembang indik kawentenan kearifan lokal basita paribasa ring pupulan tembang hits saking Dek Ulik?

Tetujon tetilik: (1) prasida nelatarang kawentenan kearifan lokal basita paribasa ring pupulan tembang hits saking Dek Ulik, (2) prasida nguningin panampen saking para yowana indik kawentenan kearifan lokal basita paribasa ring pupulan tembang hits saking Dek Ulik, Ian (3) prasida nguningin panampen saking penyanyi lan pangawi tembang indik kawentenan kearifan lokal basita paribasa ring pupulan tembang hits saking Dek Ulik.

Kawigunan pamucuk sane prasida kapolihang saking tetilik, maguna kanggen nglimbakang widya pangweruhan basa, ngicenin pangresep paajah-ajahan indik basita paribasa. Kawigunan panglimbak sane kapolihang: (1) majeng ring panilik, prasida nguningin kaajegan kearifan lokal basita paribasa ring pupulan tembang hits saking Dek Ulik. (2) majeng ring pangwacen, prasida ngawinang krama Bali utaminyane para yowana madue pikayunan nguningin utawi mlajahin basita paribasa sane pinaka kearifan lokal sane patut kaajegang, (3) majeng ring panilik lianan, kanggen pratiwimba olih panilik lianan sane madue arsa/pikayunan nglaksanayang tetilik sane mapaiketan sareng tetilik puniki, (4) majeng ring pangawi tembang, mangda sang pangawi nguningin kawentenan basita paribasa ring tembang punika dahat mawiguna sajeroning ngajegang kearifan lokal basa Bali.

Nglaksanayang tetilikan patut kadasarin saking kaweruhan sane sampun kacumpuin (Arikunto, 2005: 58). Ring tetilikan puniki nganggen makudangkudang sepat siku-siku inggih punika: (1) kearifan lokal, (2) basita paribasa, (3) tembang.

Kearifan lokal pinaka kaluwihan budaya sane tumbuh lan nglimbak ring para jana utawi krama sane kacumawisang dados wagian pinih utama lan prasida ngukuhang rasa manyama braya (Tri lan Tika, 2013: 312).

Manut Simpen (1988:5) basita paribasa teges ipun basa rinengga, punika dados rerasmen wiadin panglengut basa ri kala mababaosan kalih magegonjakan anggen pasiakranan. Wenten limolas soroh basita paribasa, inggih punika (1) sesonggan, (2) sesenggakan, wewangsalan, (4) peparikan, (5) sloka, (6) bebladbalan, (7) sesawangan, (8) pepindan, (9) cecimpelan, (10) cecangkriman, (11) cecangkitan, (12) raos ngempelin, (13) sesimbing, (14) sesemon, lan (15) sipta (Simpen, 1988: 5). 
Tembang inggih punika daging manah utawi pikenoh sang pangawi sane kasinahang majalaran antuk suara lan kruna-kruna (Jamalus lan Busroh, 1993: 14). Tembang nenten wantah kaanggen hiburan kemanten, nanging prasida kaanggen ring tradisi lan pangajahan.

Sajeroning tembang pastika sampun wenten sesuratan tembang sane ngawinang pamireng ngrasayang napi sane karasayang olih pangawi. Awe (ring Hidayat, 2014: 246) maosang sesuratan tembang pinaka semita sang angga sane sampun naanin kacingak, kapirengan utawi sane naanin karasayang.

Mapaiketan sareng tetilik puniki, tembang pop inggih punika soroh sane prasida nudut masa utawi galah santukan nginutin panglimbakan aab jagate. Tembang pop ketahnyane pinaka soroh sane ketah kaelingin miwah prasida nudut kayun sang mirengang. Wenten makulang-kulang tembang pop inggih punika tembang pop standar, tembang pop kreatif, lan tembang pop balada (Bahrudin, 2012: 2).

Panglimbak tembang pop ring Bali sayan nincap. Nyabran warsa pastika wenten penyanyi pop Bali anyar sane ngamedalang tembang Bali. Silih sinunggil penyanyi pop Bali sane kaloktah ring Bali inggih punika Dek Ulik. Dek Ulik sampun pitulas warsa dados penyanyi pop Bali. Kakawitin antuk rasa seneng matembang Bali, duk punika dane nembangang tembang Bali ring sinalih tunggil stasiun radio. Kaluwihan suaran dane sane prasida ngulangunin manah kapireng olih produser dane duk punika. Wau salanturnyane Dek Ulik kanikain sareng rekaman ring studio rekaman ring warsa kalih tali.

Dek Ulik ngawitin ngamedalang album solo saking warsa kalih tali pat ring Januadi Record. Dane sampun madue adasa album solo, inggih punika Rindu Ngantosang Janji, Sadar Beli, Somahe Mamitra, Surat Lamaran, Baju Anyar, Bli Kade, Tresna kanti Pawah, Jegeg Nyelolet, Sanjiwani, lank aping untat Langap-langap. Akehan album dane nyaritayang indik asmara, kauripan sajeroning makulawarga, miwah wenten indik agama.

\section{KRAMANING TETILIK}

Tetilik puniki ngangge tetilik deskriptif kualitatif. Tetilik deskriptif kualitatif nganggen lan ngandelang data sane masifat non verbal (tertulis). Wenten lelima papalih paridabdab kramaning tetilik sakadi: (1) palihan tetilik; palihan tetilik mapaiketan ri kala nglaksanayang tetilik. Palihan tetilik maka dasar kanggen sadurung nglaksanayang tetilik; (2) jejering lan penandang, jejering tetilik puniki pupulan tembang hits saking Dek Ulik, penandang tetiliknyane kearifan lokal basita paribasa; (3) piranti, piranti sane kanggen mupulang data inggih punika kartu data, piranti kuesioner, lan piranti sadu wicara; (4) mupulang data, mupulang data pidabdab sane nyinahang sapunapi data kapolihang. Tetilik kualitatif molihang data marupa kruna/lengkara. Kramaning kanggen mupulang data inggih punika kramaning dokumentasi, kramaning angket/kuesioner, lan kramaning sadu wicara; (5) data tureksa, pidabdab sane kalaksanayang sajeroning data tureksa sakadi reduksi data, pangwedar data, lan pacutetan utawi verifikasi.

\section{PIKOLIH LAN TETEPASAN}

Pikolih lan tetepasan kawentenan kearifan lokal basita paribasa ring pupulan tembang hits saking Dek Ulik katlatarang ring sor puniki.

Duang dasa murda tembang hits sane madaging kearifan lokal basita paribasa, inggih punika. (1) Bangkung Ngamah Gula, (2) Somahe Bebotoh, (3) Tusing Buat Tiang, (4) Ratih Kamajaya, (5) Punyan Biu, (6) Bli Kade, (7) Tresna Kanti Pawah, (8)Setrum Cadangan, (9) Suksema Beli, (10) Guru Rupaka, (11) Peteng Dedet, (12) Anak Bagus Sujati, (13) Baju Anyar, (14) Meli Sambuk di Dawan, (15) Boya ja Alpaka, (16) Ubad Rindu, (17) Buin Pidan Beli Mulih, (18) Dangap-dangap, (19) Curhat, (20) Jegeg Nyelolet. Saking duang dasa murda 
tembang punika, wantah kakeniang limang soroh basita paribasa kemanten, inggih punika sasenggakan, wewangsalan, sesawangan, peparikan, miwah sesonggan.

Pikolih kapertama inggih punika sasenggakan. Basita paribasa sasenggakan ring pupulan tembang hits saking Dek Ulik puniki kapanggihin wenten 13 imba. Kawentenan 13 imba punika kapanggihin ring 11 murda tembang, inggih punika "Bangkung Ngamah Gula", "Somahe Bebotoh", "Tusing Buat Tiang", "Ratih Kamajaya", "Punyan Biu", "Bli Kade", "Tresna Kanti Pawah", "'Setrum Cadangan", "Suksema Beli", "Guru Rupaka", "Peteng Dedet".

Imba lirik sane karanjingang ring sasenggakan sakadi "kadi I manuk kilangan pangempune, paling kemu mai inguh sing karuan", "cara ngubuh $H P$, melahang pang sing ngedrop", "beli tan bina ya kadi toyan ujane, nyiramin bungane ane sampun layu", "meme lan bapa pinaka sesuluh, sane ngicenin ajahajahaan", "kadi rasa uling ebok kanti telapakan batis", "kadi rasa kebes bibihe mamunyi", "kadi Ratih lan Semara, nemu ring pajatu karmane", "yen umpamiang kadi punyan biune kala mabuah tuah apisan", "tuturnyane alus banban, buka angin stata ngetisin", "kadi kasiramin tirta sanjiwani, galang apadang karasayang", "dadi setrum cadangan, cara ya I powerbank", "yen nirgamayang HP ngedrop, jani mara maan mecas", Ian "saja buka sasenggake layahe tanpa tulang, aluh baan beli mogbogin tiang".

Kawentenan sasenggakan ring lirik tembang hits Dek Ulik sampun yakti kariinin antuk kruna "buka". Taler wenten lirik sane kariinin antuk kruna "pinaka", "umpamiang", "kadi", miwah "cara" sane madue teges pateh sareng kruna "buka". Imba-imba lirik tembang Dek Ulik sane ngranjing ring sasenggakan taler akehan madaging sampiran lan suksemannyane.

Pikolih kaping kalih inggih punika wewangsalan. Basita paribasa wewangsalan ring pupulan tembang hits saking Dek Ulik puniki kapanggihin wenten tiga imba. Tatiga imba punika kapanggihin ring tiga murda tembang, inggih punika "Bangkung Ngamah Gula", "Anak Bagus Sujati", Ian "Baju Anyar".

Tiga imba lirik sane karanjingang ring wewangsalan minakadi "baju anyar potongan jangkung, uling Gianyar tiang ka Klungkung", "apang tusing cara mapak tebu ampasne kutang, ipidan ngarayu di tuane laut kakutang",lan "buka bangkung ngamah gula, klingkang klingkung tiang jalan mula".

Makasamian lirik sane kapanggihin ring tembang hits Dek Ulik, sane karanjingang ring wewangsalan madue lengkara kalih palet inggih punika giing lan daging, taler sampun makanti suara (a-a). Kawentenan lirik punika sampun yakti yening karanjingang ring soroh wewangsalan mawinan sampun anut sakadi teori sane katelatarang ring sepat siku-siku ngeninin wewangsalan.

Pikolih kaping tiga inggih punika sesawangan. Ring pupulan tembang hits saking Dek Ulik puniki kakeniang pitu imba lirik sane ngranjing ring sesawangan sane kapanggihin ring nem murda tembang, inggih punika "Tusing Buat Tiang", Boya ja Alpaka", "Ubad Rindu", "Tresna Kanti Pawah", "Setrum Cadangan", Ian "Buin Pidan Beli Mulih".

Imba lirik tembang sane karanjingang ring sesawangan minakadi "tanah lan langite atep rasaang tiang", ri kala tresnan iraga nedeng mabunga", beli tresna kanti gigine ba telah pawah", "panes Sang Hyang Suryane buka jani", "kangene matumpuk-tumpuk, rindune suba nagih kecag-kecog", "iang nak tunangan beli sing pemadam kebakaran", lan "ngalih gae kemu ka gumi sakura".

Lirik ring tembang hits Dek Ulik sane ngranjing ring sesawangan maosang indik napi sane kacingak lan karasayang nglantur punika kasawangang utawi kaumpamayang sakadi kahanan barang utawi palemahan. Kawentenan puniki sampun anut lan adung pisan sareng teori sesawangan sane maosang sesawangan mateges punapa-punapi ugi sane katon utawi kacingak raris karasayang ring kahyun, mirib sakadi solah kalih kahanan jadma (Simpen, 1998: 45).

Pikolih sane kaping pat inggih punika peparikan. Kawentenan soroh 
basita paribasa peparikan ring tembang hits Dek Ulik kakeniang pitung imba. Makapitu imba punika kapanggihin ring tiga murda tembang, inggih punika "Ubad Rindu", "Dangap-dangap", lan "Meli Sambuk di Dawan". Imba lirik tembang sane karanjingang ring peparikan minakadi "Gilimanuk tongos kapale malabuh, Singapadu togoge liu ditu, basang seduk dadi isinin baan bubuh, yen rindu ubadne tuah matemu", "canang sari maimbuh asepe wangi, be siap katur mawadah wanci, yening beli suba ked di rambut siwi, de engsap mareren ngaturang bakti", "apa buin legune galak-galak, ya mamilih midehan ngae barak, suba tawang legune galak-galak, Luh masare macelana bawak-bawak", "meli tanah, meli tanah meli ne aji telu tengah, ngelah somah, ngelah somah tusing taen ngoyong jumah", "meli sambuk, meli sambuk meline ditu di Dawan, beli sibuk, beli sibuk dadi anggota dewan", "tembok bata, tembok bata tembok bata warnane barak, yen makita, yen makita sinah tusing ada ajak",lan "tembok bata, tembok bata tembok bata umah Dek Ulik, yen makita, yen makita kanggoang culik-culik".

Kawentenan lirik-lirik sane ngranjing ring peparikan sampun madaging wangun-wangun sane ngwangun peparikan, minakadi kawangun antuk lengkara petang carik dados apada, lengakarane sane riinan kalih carik dados sampiran, lengkarane pungkuran sane kalih carik dados arti sajati, saha mawirama purwakanti (Simpen, 1998: 31)

Pikolih kawentenan basita paribasa kaping untat inggih punika sesonggan. Kakeniang wenten kalih imba lirik sane ngranjing ring sesonggan. Makakalih imba lirik punika kapanggihin ring kalih murda tembang inggih punika "Curhat", lan "Jegeg Nyelolet".

Imba lirik tembang sane ngranjing ring sesonggan minakadi "basange dawanang", lan "turmaning pang sing ngamik di margane". Makakalih imba lirik sane karanjingang ring sesonggan punika sampun kaanutang sareng teori sesonggan manut Simpen (1998: 6), sane maosang kruna sasonggan puniki dados ngintar basa utawi kata ungkapan, artos ipun tatakan (penyangga) kruna sane dados panglengut basa. Saking makakalih imba lirik punika, sampun sinah kawentenannyane kaanggen panglengut basa utawi pemanis basa mangda punapa-punapi sane sujatinyane meled kabaosang prasida kapireng alus yening saihang ring arti sujatinyane.

Pikolih lan tetepasan saking panampen para yowana ngeninin indik kawentenan kearifan lokal basita paribasa ring pupulan tembang hits saking Dek Ulik katlatarang ring sor puniki.

Panampen saking para yowana puniki kapolihang majalaran antuk nganggen kramaning kuesioner. Kuesioner puniki kawedar majeng ring angga jadma sane yusanyane kantun kabaos yowana, inggih punika angga saking sisia SMA, angga saking mahasisia, lan angga saking sekaa trunatruni. Saking satus kuesioner sane sampun kawedar majeng ring para yowana, kapolihang panampen sane malianan pantaraning soang-soang yowana. Panilik ngwedar kuesioner sane madaging nem parindikan sane kaicen panampen olih para yowana, inggih punika panampen pinih cumpu, cumpu, kirang cumpu, lan nenten cumpu. Saking pikolih kuesioner punika, panilik prasida nyutetang panampen para yowana ngeninin indik kawentenan kearifan lokal basita paribasa ring pupulan tembang hits saking Dek Ulik.

Parindikan sane kapertama sane kaunggahang inggih punika kawentenan basita paribasa sane kauningin utawi polih kaplajahin saking sekolah lan genah tiosan. Kaping kalih, maosang indik basita paribasa sane dangan karesepang. Parindikan sane kaping tiga maosang indik mabebaosan nganggen basita paribasa sarahina. kaping pat inggih punika sering mirengang tembang pop Bali minakadi tembang saking Dek Ulik. 
Kaping lima ngeninin indik kawentenan basita paribasa ring tembang pop Bali (tembang hits saking Dek Ulik). Kaping untat ngeninin indik basita paribasa ring tembang pop Bali (tembang Dek Ulik) pinaka silih sinunggil piranti ngajegang kearifan lokal basita paribasa ring Bali.

Saking nem parindikan sane kaunggahang ring kuesioner, panilik prasida nyutetang panampen saking para yowana ngeninin indik kawentenan kearifan lokal basita paribasa ring pupulan tembang hits Dek Ulik. Para yowana ngicenin panampen sane positif utawi becik ngeninin indike punika. Yadiastun marasa meweh ngresepang teges miwah soroh-soroh basita paribasa, nanging para yowana kantun eling tekening kawentenan basita paribasa punika. Mawinan sampun polih mlajahin daweg saking SD. Para yowana taler sering mirengang tembang Dek Ulik, dadosnyane prasida uning tekening kawentenan basita paribasa ring pupulan tembang hits Dek Ulik. Kawentenan punika kacumpuin prasida kadadosang piranti ngajegang kearifan lokal basita paribasa.

Pikolih lan tetepasan saking panampen penyanyi lan sinalih tunggil pangawi tembang ngeninin indik kawentenan kearifan lokal basita paribasa ring pupulan tembang hits saking Dek Ulik katlatarang ring sor puniki.

Mapaiketan sareng kawentenan pupulan tembang hits Dek Ulik sane katilikin ring tetilikan puniki, dadosnyane kabuatang pisan panampen saking Dek Ulik pinaka sang sane nembangang lan sang pangawi pinaka sang sane ngawi tembang-tembang hits punika. Kawentenan basita paribasa ring pupulan tembang hits Dek Ulik nenten wantah patut kauningin olih para yowana. Penyanyi sane maktayang tembang, lan pangawi sane ngawi tembang, sampun pinih utama patut nguningin lirik tembang sane madaging basita paribasa. Mawinan dane makakalih sane kapertama prasida uning lan ngresepin tembang punika wau salanturnyane kapiarsayang olih krama lianan. Yakti sampun ring tetilikan puniki mabuat pisan nguningin panampen saking penyanyi lan pangawi tembang indik kawentenan kearifan lokal basita paribasa ring tembang druenyane. Penampen saking penyanyi lan sinalih tunggil pangawi tembangnyane kapolihang antuk ngamargiang kramaning sadu wicara.

Kapertama panilik sampun ngamargiang sadu wicara sareng Dek Ulik kaanggen nyangkepin data panampen saking penyanyi. Akeh pitaken sane sampun katakenang ngeninin indik pamargin dane dados penyanyi lan pitaken kaanggen nyawis bantang pikobet. Wenten lima pitaken sane sampun katakenang kaanggen nyawis bantang pikobet, inggih punika panampen Dek Ulik ngeninin indik kawentenan kearifan lokal basita paribasa ring pupulan tembang hits druenyane.

Dane maosang uning indik kawentenan basita paribasa ring lirik tembangnyane. Yadiastun dane nenten tatas uning tekening soroh basita paribasa, nanging indik teges basita paribasa ring lirik tembangnyane sampun prasida kauningin. Mawinan ring tembangnyane punika taler katelatarang indik tegesnyane. Manut dane, sampun patut pisan yening uning tekening daging tembang, mangda prasida becik ri kala maktayang tembang punika.

Ngeninin indik kawigunan basita paribasa ring tembang, dane ngicenin panampen basita paribasa punika prasida ngawinang para pamiarsa meled uning indik tembang punika. Dadosnyane dane ngicenin panampen becik pisan indik kawentenan basita paribasa ring tembangnyane. Manut dane, mangkin sampun sayan akeh krama Bali sane seneng mirengang tembang pop Bali. Kawentenan puniki prasida kadadosang sarana ngajegang basa Bali inggih punika basita paribasa. Napi malih ring aab mangkin sampun mabinayan sareng ipidan. Yening ipidan kantun kentel antuk kabudayaan. Ring aab sakadi mangkin sampun akeh krama Bali sane gengsi ngicenin okanyane paplajahan mabasa Bali, saking embas sampun kaajahin mabebaosan nganggen basa Indonesia kemanten. Kaping untat, dane maosang cumpu pisan yening kawentenan tembang pop Bali puniki kadadosang sarana ngajegang basita paribasa sane pinaka kearifan lokal basa Bali. 
Salanturnyane sadu wicara sareng A.A. Raka Sidan taler sampun kamargiang. A.A. Raka Sidan maosang nenten uning makasamian soroh basita paribasa. Nanging dane pinih uning indik kawentenan basita paribasa ring tembang kawiannyane. Ri kala ngawi tembang sane madaging basita paribasa, ketahnyane dane ngangge basita paribasa sane anyar sane kaanutang sareng kawigunannyane sajeroning tembang. Nanging dane taler nganggen basita paribasa sane sampun wenten saking dumun sane nenten populer lan mangkin sampun prasida kauningin olih krama majalaran antuk tembang kawiannyane.

Dane taler maosang, kawentenan basita paribasa sajeroning tembang prasida kadadosang sinalih tunggil sarana anggen mlajahin basita paribasa, yadiastun kawentenannyane nenten akeh. Napi malih ring aab mangkin rasa bangga mabasa Bali punika sayan padem. Tembang pop Bali sane sayan warsa sayan nglimbak, sayan kasenengin olih krama Bali prasida dados sarana ngajegang basa Bali, kenten taler basita paribasa.

Dadosnyane tembang pop Bali wantah sinalih tunggil piranti kaanggen ngajegang basa lan budaya Bali. Salanturnyane patut makasamian krama Bali sane sareng-sareng madue pikayunan ngajegang budaya Bali. Dane ngicenin panampen minab nenten makasamian soroh basita paribasa prasida kaanggen ring tembang kawiannyane Nanging sakirangnyane prasida kaanggen sarana nguningin basita paribasa.

Tetilik ngeninin indik basita paribasa sujatinnyane sampun kalaksanayang olih panilik lianan. Mawit saking kawentenane punika panilik nyaihang pikolih lan tetepasan tetilik puniki sareng tetilik panilik lianan, sakadi: (1) tetilik saking I Nyoman Artawa sane mamurda "Basita Paribasa ring Album Mabalih Wayang Pakardin Ketut Bimbo". Jejering ring tetilikan dane sampun pastika malianan sareng tetilikan panilik. Dane nganggen album Mabalih Wayang pakardin Ketut Bimbo, panilik nganggen pupulan tembang pop Bali hits olih Dek Ulik. Ring penandang tetilikan dane pateh tekening tetilikan panilik, inggih punika maosang indik basita paribasa. Nanging ring tetilikan puniki madaging kearifan lokal sumangdene kauningin basita paribasa punika rumasuk kearifan lokal Bali. Tetilik puniki taler madue kaluwihan mawinan nganggen parikrama sadu wicara lan kuesioner sane pacang ngawinang pikolih tetilikan puniki jangkep.

(2) tetilikan pakaryan Gusti Ayu Era Aryanti. Tetilik dane mamurda Seseleh Basita Paribasa lan Kawentenan Guna Sarat ring Daging Cakepan Drama Nang Kepod Pakardin Ketut Aryana. Tetilik dane madue pabinayan sareng tetilik puniki. Pabinaannyane wantah ring penanlang lan jejering tetilik. Ring penanlang, dane nganggen kalih variabel inggih punika basita paribasa lan guna sarat. Ring tetilik puniki wantah ngangge siki variabel inggih punika kearifan lokal basita paribasa. Ngeninin indik jejering tetilik, dane ngangge Daging Cakepan Drama Nang Kepod Pakardin Ketut Aryana. Dane ngangge kasusastraan drama kadadosang jejering. Nanging panilik ngangge Pupulan Tembang Pop Bali Hits Olih Dek Ulik.

Tetilikan dane sampun becik mawinan akeh molihang soroh-soroh basita paribasa ring cakepan drama punika. Ring tetilik puniki nenten akeh molihang soroh-soroh basita paribasa sakadi ring cakepan drama. Mawinan nenten makasamian soroh basita paribasa becik kadagingin ring tembang.

Yadiastun asapunika, tetilikan puniki madue kaluwihan yening kasandingang sareng tetilikan dane. Tetilikan puniki nilikin indik tembang sane pastika pamiletnyane akeh tur kasenengin olih krama Bali. Majanten sampun akeh sane pacang meled ngwacen tur nguningin daging tetilikan puniki. Napi malih tetilikan puniki nganggen kramaning sadu wicara sareng Dek Ulik miwah sinalih tunggil pangawi saking tembang dane.

Tetilikan puniki madue kawigunan ri sajeroning ngajengang kearifan lokal basita paribasa. Saking tetilikan puniki, para jana pacang nguningin lan prasida mlajahin indik basita paribasa sane pinaka kearifan lokal Bali. Tetilikan puniki prasida 
ngicenin kaweruhan mabebaosan ngangge basita paribasa.

Sangkaning asapunika, sasampune ngwacen tetilikan puniki kaaptiang sang pangwacen sida ngresep. Para jana taler pacang nguningin kawentenan utsaha ngajegang basita paribasa ring pupulan tembang saking Dek Ulik puniki, sane salanturnyane kaaptiang mangda para jana nyarengin ngajegang kearifan lokal basita paribasa.

\section{PAMUPUT}

Manut pikolih lan tetepasan tetilik sane sampun katlatarang, prasida kacutetang kawentenan basita paribasa ring pupulan tembang hits saking Dek Ulik. Saking 20 murda tembang, kapolihang wenten 5 soroh basita paribasa saking 15 soroh basita paribasa sane kaanggen ring sepat siku-siku. Taler indik akehnyane kakeniang wenten 31 imba. Soroh basita paribasa punika sakadi sasenggakan kakeniang 13 imba, wewangsalan kakeniang 3 imba, sesawangan kakeniang 7 imba, peparikan kakeniang 7 imba, lan sesonggan sane kakeniang wenten 2 imba. Dadosnyane kawentenan soroh basita paribasa sasenggakan pinih akeh kapanggihin ring pupulan tembang hits saking Dek Ulik

Saking nem parindikan sane kaunggahang ring kuesioner, prasida kacutetang para yowana ngicenin panampen sane positif utawi becik ngeninin indik kawentenan kearifan lokal basita paribasa ring pupulan tembang hits saking Dek Ulik. Yadiastun marasa meweh ngresepang teges miwah sorohsoroh basita paribasa, nanging para yowana kantun eling tekening kawentenan basita paribasa punika. Mawinan sampun polih mlajahin daweg saking SD. Para yowana taler sering mirengang tembang Dek Ulik, dadosnyane prasida uning tekening kawentenan basita paribasa ring pupulan tembang hits Dek Ulik. Kawentenan punika kacumpuin prasida kadadosang piranti ngajegang kearifan lokal basita paribasa.

Saking sadu wicara sane sampun kamargiang majeng ring Dek Ulik pinaka penyanyi lan A.A. Raka Sidan pinaka pangawi tembang, kapolihang panampen dane makakalih indik kawentenan kearifan lokal basita paribasa ring pupulan tembang hits saking Dek Ulik. Pamicutetnyane dane makakalih pinaka seniman Bali cumpu yening kawentenan tembang pop Bali prasida ngawinang krama Bali eling ring basa Bali. Kenten taler kawentenan basita paribasa sane kajangkepin antuk tegesnyane ring tembang dane prasida ngawinang krama Bali nenten lali tekening kawentenan basita paribasa. Yadiastun nenten makasamian soroh basita paribasa prasida kaanggen ring tembang dane, nanging sakirangnyane prasida kaanggen sarana nguningin basita paribasa.

Piteket kawedarang majeng ring: (1) krama Bali, utaminnyane para yowana kaaptiang mangda eling lan uning tekening kawentenan basita paribasa ring tembang pop Bali, inggih punika ring pupulan tembang hits saking Dek Ulik. Kawentenan punika mangda kadadosang dasar ring para yowana ri kala mlajahin lan nincapang kawagedan mabasita paribasa.

(2) majeng ring guru lan dosen pinaka sang sane ngicenin pangajahan, mangda kawentenan puniki kaanggen sarana ri kala ngicenin pangajahan indik basita paribasa. Mawinan yening mlajahin basita paribasa nganggen piranti tembang, napi malih tembang pop Bali sane kasenengin, pastika nenten ngawinang rasa med ri kala mlajahin.

(3) majeng ring pangawi tembang kaaptiang mangda prasida kaanggen pratiwimba lan sasuluh majeng ring pangawi tembang ring album puniki. Mangda pangawi sayan nglimbakang kawagedannyane sajeroning ngarya tembang sane madaging basita paribasa. Taler mangda sang pangawi nguningin kawentenan basita paribasa ring tembang punika dahat mawiguna sajeroning ngajegang kearifan lokal basa Bali.

(4) majeng ring panilik lianan kaaptiang mangda prasida nglimbakang tetilik puniki. Panglimbak sane kaaptiang inggih punika mangda nglaksanayang tetilik sane asoroh lan prasida maripurnayang tetilikan puniki mangda 
sayan mawiguna sajeroning nglimbakang kawentenan basa Bali.

\section{KAPUSTAKAAN}

Agustiana, I Gusti Ayu Tri lan I Nyoman Tika. 2013. Konsep Dasar IPA. Yogyakarta: Penerbit Ombak.

Arikunto, Suharismi. 2005. Manajemen Penelitian. Jakarta: PT Rineka Cipta.

Artawa, I Nyoman. 2016. Basita Paribasa ring Album Mabalih Wayang Pakardin Ketut Bimbo. Skripsi (nenten kamijilang). Jurusan Pendidikan Bahasa Bali, Undiksha.

Aryanti, Gusti Ayu Era. 2015. Seseleh Basita Paribasa lan Kawentenan Guna Sarat ring Daging Cakepan Drama Nang Kepod Pakardin Ketut Aryana. Skripsi (nenten kamijilang). Jurusan Pendidikan Bahasa Bali, Undiksha.
Bahrudin. 2012. Ciri-ciri Musik Pop, (Online), (http://musikmajid.blogspot.com/201 2/12/ciri-ciri-musik-pop.html, diakses 16 Januari 2017).

Chaer, Abdul. 1994. Linguistik Umum. Jakarta: Rineka Cipta.

Hidayat, Rahmat. 2014. Analisis Semiotika Makna Motivasi pada Lirik Lagu "Laskar pelangi" Karya Nidji. eJournal IImu Komunikasi, (Online), 2 (1): 243-258, (http://ejournal.ilkom.fisipunmul.ac.id, diakses 15 Januari 2017).

Jamalus, Hamzah Busroh. 1993. Pendidikan Kesenian 1 (Musik). Jakarta: Departemen Pendidikan dan Kebudayaan Direktorat Jenderal Pendidikan Tinggi Proyek Pembinaan Tenaga Kependidikan.

Simpen, I Wayan. 1988. Basita Paribasa. Denpasar: PT. Upada Sastra. 